\title{
APPROXIMATE WEAK AMENABILITY OF ABSTRACT SEGAL ALGEBRAS
}

\author{
H. SAMEA
}

\begin{abstract}
In this paper the approximate weak amenability of abstract Segal algebras is investigated. Applications to compact groups are given. Also an open problem raised by Ghahramani and Lau is answered negatively.
\end{abstract}

\section{Introduction}

In [3], Ghahramani and Lau studied approximate weak amenability of Segal algebras on locally compact groups. We continue the study of the notions approximate weak amenability for a larger class of Banach algebras that called abstract Segal algebras.

The organization of this paper is as follows. Section 1 is devoted to preliminaries and notations which are needed throughout the rest of the paper. In Section 2 the structure of abstract Segal algebras is studied. Also the approximate weak amenability of this class of Banach algebras is investigated. Section 3 is devoted to application of section 2 to convolution Banach algebras on compact groups. It is shown that if $G$ is an infinite compact abelian group, then the symmetric Segal algebras $A(G)$ and $L^{2}(G)$ are not approximately amenable. This establishes negatively the Open Question 2 of [3]. Furthermore, it is proved that if $G$ is a compact group, then for the abstract Segal subalgebra $L^{\infty}(G)$ of $L^{1}(G)$, the notions of approximate weak amenability, and weak amenability are equivalent to finiteness of $G$. This contradicts Remark 3.4 of [3]. Indeed, if $G$ is an infinite compact group, then $L^{\infty}(G)$ is an abstract Segal subalgebra of the amenable Banach algebra $L^{1}(G)$ that is not approximately weakly amenable.

\section{Preliminaries}

Let $A$ be a Banach algebra, and let $B$ and $C$ be non-empty subsets of $A$. Define $B . C=\{b c: b \in B, c \in C\}$, and $B C$ to be the linear span of B.C. Write $B^{2}$ for $B B$. 
Let $A$ be a Banach algebra, and let $X$ be a Banach $A$-bimodule. A left approximate identity in $A$ for $X$ is a net $\left(e_{\alpha}\right)$ in $A$ such that for each $x \in X$, $\lim _{\alpha} e_{\alpha} \cdot x=x$. Right approximate identity and approximate identity in $A$ for $X$ are defined similarly. A bounded linear map $D: A \rightarrow X$ is called an $X$-derivation, if

$$
D(a b)=D(a) \cdot b+a \cdot D(b) \quad(a, b \in A) .
$$

For example, take $x \in X$, and set $a d_{x}(a)=a . x-x . a(a \in A)$. Then $a d_{x}$ is a derivation; such derivations are termed inner derivations. A derivation $D: A \rightarrow X$ is approximately inner if there exists a net $\left(x_{\alpha}\right) \subseteq X$ such that for every $a \in A, D(a)=\lim _{\alpha} a d_{x_{\alpha}}(a)$. A Banach algebra $A$ is amenable (approximately amenable, respectively) if every derivation from $A$ into $X^{*}$ is inner (approximately inner, respectively) for all Banach $A$-bimodules $X$. For more details see [5] and [8].

Every Banach algebra $A$ with the product of $A$ giving the two module multiplications defines a Banach $A$-bimodule. Let $A^{*}$ be the dual $A$-bimodule. A Banach algebra $A$ is weakly amenable if each derivation from $A$ into $A^{*}$ is inner. Recall from [3] that a Banach algebra $A$ is approximately weakly amenable if each derivation from $A$ into $A^{*}$ is approximately inner.

Let $\left(A,\|\cdot\|_{A}\right)$ be a Banach algebra. Recall from [3], a Banach algebra $\left(B,\|\cdot\|_{B}\right)$ is an abstract Segal subalgebra of $A$ if:

(i) $B$ is a dense left ideal in $A$.

(ii) There exists $M>0$ such that $\|b\|_{A} \leq M\|b\|_{B}$ for each $b \in B$.

(iii) $\|a b\|_{B} \leq\|a\|_{A}\|b\|_{B}(a, b \in B)$.

Let $G$ be a locally compact group. A Banach space $\left(S(G),\|\cdot\|_{S(G)}\right)$ is Segal algebra on $G$ if:

(i) $S(G)$ is dense in $L^{1}(G)$.

(ii) There exists $M>0$ such that $\|f\|_{1} \leq M\|f\|_{S(G)}$ for all $f \in S(G)$.

(iii) $S(G)$ is left translation invariant, $\left\|\delta_{x} * f\right\|_{S(G)}=\|f\|_{S(G)}(f \in S(G)$, $x \in G)$, and the map $x \mapsto \delta_{x} * f$ from $G$ into $S(G)$ is continuous for all $f \in S(G)$.

That a Segal algebra on $G$ is an abstract Segal algebra is well-known; see [1], page 492.

A Segal algebra $S(G)$ is symmetric if it is right translation invariant, and for all $f \in S(G),\left\|f * \delta_{x}\right\|_{S(G)}=\|f\|_{S(G)}(x \in G)$, and the map $x \mapsto f * \delta_{x}$ from $G$ into $S(G)$ is continuous for all $f \in S(G)$. 


\section{Approximate weak amenability of abstract Segal algebras}

We start this section with the following.

Lemma 2.1. Let $A$ be a Banach algebra with a bounded left approximate identity, $B$ an abstract Segal subalgebra of A. Then A.B is an abstract Segal subalgebra of $A$, and $A . B=\overline{A B}^{\|\cdot\|_{B}}$. Furthermore, there exists a left approximate identity for A.B which is also a bounded left approximate identity for A.

Proof. It is easy to check that $\overline{A B} \|^{\|\cdot\|_{B}}$ is a Banach left $A$-module. Let $\left(e_{\alpha}\right)$ be a bounded left approximate identity for $A$. Let $x \in \overline{A B}{ }^{\|\cdot\|_{B}}$. Suppose $\delta>0$. There exists $x_{\delta}=\sum_{i=1}^{n} a_{i} b_{i} \in A B$, where $n \in \mathrm{N}, a_{i} \in A$, and $b_{i} \in B$ $(1 \leq i \leq n)$, such that $\left\|x-x_{\delta}\right\|_{B}<\delta$. Applying the triangular inequality and properties of $B$, we have

$$
\left\|e_{\alpha} x-x\right\|_{B} \leq\left(1+\sup _{\alpha}\left\|e_{\alpha}\right\|_{A}\right) \delta+\sum_{i=1}^{n}\left\|e_{\alpha} a_{i}-a_{i}\right\|_{A}\left\|b_{i}\right\|_{B} .
$$

Hence $\lim \sup _{\alpha}\left\|e_{\alpha} x-x\right\|_{B} \leq\left(1+\sup _{\alpha}\left\|e_{\alpha}\right\|_{A}\right) \delta$, and so $\left(e_{\alpha}\right)$ is a bounded approximate left identity in $A$ for $\overline{A B}^{\|\cdot\|_{B}}$. By Cohn's Factorization Theorem (Theorem 32.23 of [7]) $\overline{A B}^{\|\cdot\|_{B}}=A . B$. Let $a \in A$, and $\epsilon>0$. There exists $b_{\epsilon} \in B$ such that $\left\|a-b_{\epsilon}\right\|_{A}<\frac{\epsilon}{2}$. Also, there exists $\alpha$ such that $\left\|b_{\epsilon}-e_{\alpha} b_{\epsilon}\right\|_{A}<$ $\frac{\epsilon}{2}$. Hence $\left\|a-e_{\alpha} b_{\epsilon}\right\|_{A}<\epsilon$. Since $e_{\alpha} b_{\epsilon} \in A . B, A . B$ is dense in A. Hence A.B is an abstract Segal subalgebra of $A$.

For each $\epsilon>0$ and $\alpha$, there exists $e_{(\alpha, \epsilon)} \in A . B$ such that $\left\|e_{\alpha}-e_{(\alpha, \epsilon)}\right\|_{A} \leq$ $\min \{\epsilon, 1\}$. By a simple calculation one can prove that $\left(e_{(\alpha, \epsilon)}\right)_{(\alpha, \epsilon)}$ is a bounded left approximate identity for $A$. Since for $a \in A$, and $b \in B, \| e_{(\alpha, \epsilon)}(a b)-$ $(a b)\left\|_{B} \leq\right\| e_{(\alpha, \epsilon)} a-a\left\|_{A}\right\| b \|_{B},\left(e_{(\alpha, \epsilon)}\right)$ is a left approximate identity for $A . B$.

Similar to Theorem 2.8.63 of [1], we have the following result.

Proposition 2.2. If $A$ is an approximately weakly amenable Banach algebra, then $\overline{A^{2}}=A$.

Proof. If $\overline{A^{2}} \neq A$, then there exists $a_{0} \in A \backslash \overline{A^{2}}$. By the Hahn-Banach theorem there exists $\phi \in A^{*}$ such that $\phi_{\mid \overline{A^{2}}}=0$ and $\phi\left(a_{0}\right)=1$. It is easy to show that the map $D: A \rightarrow A^{*}, a \mapsto \phi(a) \phi$ is a derivation. Since $D\left(a_{0}\right)\left(a_{0}\right)=1$ and for each net $\left(\xi_{\alpha}\right) \subseteq A^{*}, \lim _{\alpha}\left(a_{0} \cdot \xi_{\alpha}-\xi_{\alpha} \cdot a_{0}\right)\left(a_{0}\right)=0$, we have a contradiction.

Theorem 2.3. Let $A$ be a Banach algebra with a bounded approximate left identity, and $B$ be an abstract Segal subalgebra of A. If $B$ is approximately 
weakly amenable, then $B=A . B$, and $B$ has an approximate left identity that is also a bounded approximate left identity for $A$.

Proof. By Proposition 2.2, and Lemma 2.1

$$
B={\overline{B^{2}}}^{\|\cdot\|_{B}} \subseteq \overline{A B}^{\|\cdot\|_{B}}=A \cdot B \subseteq B .
$$

Therefore $B=A . B$. Now, by Lemma 2.1 there exists an approximate left identity for $B=A$. $B$ which is also a bounded approximate left identity for $A$. Hence $\left(e_{\alpha}\right)$ is an approximate identity for $B$.

Definition 2.4. Let $\left(A,\|\cdot\|_{A}\right)$ be a Banach algebra. A Banach algebra $\left(B,\|\cdot\|_{B}\right)$ is a symmetric abstract Segal algebra of $A$ if:

(i) $B$ is a dense ideal in $A$.

(ii) There exists $M>0$ such that $\|b\|_{A} \leq M\|b\|_{B}$ for each $b \in B$.

(iii) $\|a b\|_{B},\|b a\|_{B} \leq\|a\|_{A}\|b\|_{B}(a, b \in B)$.

Lemma 2.5. Let $A$ be a Banach algebra with a bounded approximate identity, $B$ a symmetric abstract Segal subalgebra of A. Then A.B.A is a symmetric Segal algebra of $A$ such that there exists an approximate identity for A.B.A which is also a bounded approximate identity for A.

Proof. It is easy to check that $\overline{A B A} \|^{\|\cdot\|_{B}}$ is a Banach $A$-bimodule. Let $\left(e_{\alpha}\right)$ be a bounded approximate identity for $A$. Let $\left(e_{(\alpha, \epsilon)}\right)$ be as the proof of Lemma 2.1. By a similar way as the proof of Lemma 2.1 , it can be proved that $\overline{A B A} \|^{\|\cdot\|_{B}}=$ $A . B . A$, and $\left(e_{(\alpha, \epsilon)}\right)$ is an approximate identity for the Banach algebra A.B.A. The remainder is proved in a similar manner.

THeorem 2.6. Let $A$ be a Banach algebra with a bounded approximate identity, $B$ a symmetric abstract Segal subalgebra of $A$. If $B$ is approximately weakly amenable, then $B=A . B . A$, and $B$ has an approximate identity that is also a bounded approximate identity for $A$.

Proof. By Proposition 2.2, $\bar{B}^{\left\|^{\|} \cdot\right\|_{B}}=B$. From this fact, Lemma 2.1, and Lemma 2.5

$$
\begin{aligned}
& B={\overline{B^{2}}}^{\|\cdot\|_{B}}={\overline{\bar{B}^{2}}}^{\|\cdot\|_{B}}\left\|^{\|\cdot\|_{B}} \subseteq \overline{\overline{A B}}^{\|\cdot\|_{B}} A\right\|^{\|\cdot\|_{B}} \\
& =\overline{(A . B) A}^{\|\cdot\|_{B}} \subseteq \overline{A B A}^{\|\cdot\|_{B}}=A \cdot B \cdot A \subseteq B .
\end{aligned}
$$

Therefore $B=A . B . A$. Now, by Lemma 2.5 there exists an approximate identity for $B=A . B . A$ which is also a bounded approximate identity for $A$.

It seems that the hypothesis " $A$ is a symmetric abstract Segal algebra, and $B$ has an approximate identity" in Remark 3.4 of [3] is omitted. The following 
theorem is a correct version of Remark 3.4 of [3] with its inverse. In the next chapter, an example is given to show that the hypothesis " $B$ has an approximate identity" can not be removed.

TheOREm 2.7. Let A be an amenable Banach algebra, B a symmetric abstract Segal algebra of $A$. Then the following statements are equivalent:

(i) $B$ is approximately weakly amenable.

(ii) $B$ has an approximate identity.

Proof. (i) $\Rightarrow$ (ii): Since $A$ is amenable, so by Proposition 2.2.1 of [8] it has a bounded approximate identity. Hence by Theorem 2.6, $B$ has an approximate identity.

(ii) $\Rightarrow$ (i): The proof is the same as that of Theorem 3.1 of [3].

COROLlary 2.8. Let $G$ be an amenable locally compact group, $S(G)$ a symmetric abstract Segal subalgebra of $L^{1}(G)$. Then $S(G)$ is approximately weakly amenable, if and only if, $S(G)$ has an approximate identity.

Proof. Note that by Johnson's Theorem (Theorem 2.1.8 of [8]), $L^{1}(G)$ is amenable.

Remark 2.9. Let $G$ be a locally compact group, and $S(G)$ be an approximately weakly amenable abstract Segal subalgebra of $L^{1}(G)$, that is also left invariant, and $\left\|\delta_{x} * f\right\|_{S(G)}=\|f\|_{S(G)}$ for all $x \in G$ and $f \in S(G)$. Then from Theorem 2.3, $S(G)=L^{1}(G) * S(G)$. Hence $S(G)$ is a Segal algebra on $G$ (note that the continuity of $x \mapsto \delta_{x} * f ; G \rightarrow S(G)$, where $f \in S(G)$, is follows from the inequality

$\left\|\delta_{x} *(g * h)-g * h\right\|_{S(G)} \leq\left\|\delta_{x} * g-g\right\|_{1}\|h\|_{S(G)} \quad\left(g \in L^{1}(G), h \in S(G)\right)$, and Theorem 20.4 of [6]).

\section{Approximate weak amenability of convolution Banach algebras on compact groups}

The following Proposition establishes negatively the Open Question 2 of [3].

Proposition 3.1. Let $G$ be an infinite, abelian, compact group with the normalized Haar measure. Then the convolution Banach algebras $L^{2}(G)$ and $A(G)$ are symmetric Segal algebras on $G$ that are not approximately amenable.

Proof. Let $\widehat{G}$ be the set of all equivalence classes of continuous irreducible representations of $G$ (see section 27 of [7]). By Remarks 27.4(i) of [7], each $\pi \in \widehat{G}$ is one-dimensional. Also, by Lemma 28.1 of [7], $\widehat{G}$ is infinite. Let $1 \leq p<\infty$. Since $\widehat{G}$ is infinite, so there exists a continuous epimorphism 
from the Banach algebra $\ell^{p}(\widehat{G})$ with point-wise multiplication into the Banach algebra $\ell^{p}(\mathrm{~N})$ with point-wise multiplication. Hence by Theorem 4.1 of [2] and Proposition 2.2 of [5], $\ell^{p}(\widehat{G})$ is not approximately amenable. By the PeterWeyl theorem the convolution Banach algebra $L^{2}(G)$ is isometrically algebra isomorphic with the Banach algebra $\ell^{2}(\widehat{G})$ with point-wise multiplication, and hence is not approximately amenable. From Theorem 34.35 of [7], the convolution Banach algebra $A(G)$ is isometrically algebra isomorphic with the Banach algebra $\ell^{1}(\widehat{G})$ with point-wise multiplication, and hence is not approximately amenable.

Let $G$ be a compact group. It is evident that the convolution Banach algebra $L^{\infty}(G)$ is a symmetric abstract symmetric Segal subalgebra of $L^{1}(G)$.

Proposition 3.2. Let $G$ be a compact group. Then for the convolution Banach algebra $L^{\infty}(G)$, the notions of approximate weak amenability, and weak amenability are equivalent to finiteness of $G$.

Proof. It is clear that if $G$ is finite, then $L^{\infty}(G)$ is weakly amenable.

Assume that $L^{\infty}(G)$ is approximately weakly amenable. By Theorem 20.16 of [6], and Theorem 2.3, $L^{\infty}(G)=L^{1}(G) * L^{\infty}(G) \subseteq C(G)$. Now, by Lemma 37.3 of [7], $G$ is finite.

Remark 3.3. By the above proposition, if $G$ is an infinite compact group, then the symmetric abstract Segal algebra $L^{\infty}(G)$ is not approximately weakly amenable. So Remark 3.4 of [3] are not valid for all abstract Segal subalgebras of $L^{1}(G)$, even if $G$ is amenable.

Let $G$ be a compact group with the normalized Haar measure. By Corollary 2.8 (see also [3]), the convolution Banach algebras $L^{p}(G)(1<p<\infty)$ are approximately weakly amenable. In the following proposition, the weak amenability of the convolution Banach algebras $L^{p}(G)(2 \leq p<\infty)$ is studied.

Proposition 3.4. Let $G$ be a compact group and $2 \leq p<\infty$. Then the convolution algebra $L^{p}(G)$ is weakly amenable if and only if $G$ is abelian or finite.

Proof. If $G$ is abelian, then by Corollary 3.4 of [4] the convolution Banach algebra $L^{p}(G)$ is weakly amenable. If $G$ is finite, then $\ell^{p}(G)\left(=\ell^{1}(G)\right)$ is not only weakly amenable, but also amenable.

If $G$ is an infinite non-abelian compact group, then there exist $x, y \in G$ such that $x y \neq y x$. Since $2 \leq p<\infty$, so if $\frac{1}{p}+\frac{1}{q}=1$, then $p \geq q$. Hence $L^{p}(G) \subseteq L^{q}(G)$. It follows that the mapping

$$
D_{x}: L^{p}(G) \rightarrow L^{q}(G), \quad f \mapsto \delta_{x} * f-f * \delta_{x},
$$


defines a well-defined derivation. By a similar method as Remark 3.2 of [4], we conclude that $D_{x}$ is not inner. It is easy to check that the well-known isometrical Banach-space isomorphism $T: L^{q}(G) \rightarrow L^{p}(G)^{*}$, that given by

$$
\langle g, T(f)\rangle=\int_{G} f(x) g\left(x^{-1}\right) d x \quad\left(f \in L^{q}(G), g \in L^{p}(G)\right),
$$

defines an $L^{p}(G)$-module homomorphism. It follows that the convolution Banach algebra $L^{p}(G)$ is not weakly amenable.

Question: Is the conclusion of the Proposition 3.4 valid for each $1<$ $p<2$ ? Note that it is well known that for each locally compact group, the group algebra $L^{1}(G)$ is weakly amenable. Also, in Propositions 3.2 and 3.4, a necessary and sufficient condition for weak amenability of Banach algebras $L^{p}(G)(2 \leq p \leq \infty)$ is found.

ACKNOWLEDGEMENT. The author would like to thank the referee for invaluable comments. The author also would like to thank the University of Bu-Ali Sina (Hamedan) for its support.

\section{REFERENCES}

1. Dales, H. G., Banach Algebras and Automatic Continuity, London Math. Soc. Monographs 24, Clarendon Press, Oxford Univ. Press, New York 2000.

2. Dales, H. G., Loy, R. J., and Zhang, Y., Approximate amenability for Banach sequence algebras, Studia Math. 177 (2006), 81-96.

3. Ghahramani, F., and Lau, A. T.-M., Approximate weak amenability, derivations and Arens regularity of Segal algebras, Studia Math. 169 (2005), 189-205.

4. Ghahramani, F., and Lau, A. T.-M., Weak amenability of certain classes of Banach algebras without bounded approximate identities, Math. Proc. Cambridge Philos. Soc. 133 (2002), 357-371.

5. Ghahramani, F., and Loy, R. J., Generalized notions of amenability, J. Funct. Anal. 208 (2004), 229-260.

6. Hewitt, E. and Ross, K. A., Abstract Harmonic Analysis, Vol. I, 2nd ed., Grundlehren der math. Wissenschaften 115, Springer, Berlin 1979.

7. Hewitt, E., and Ross, K. A., Abstract Harmonic Analysis, Vol. II, Grundlehren der math. Wissenschaften 152, Springer, Berlin 1970.

8. Runde, V., Lectures on Amenability, Lecture Notes in Mathematics 1774, Springer, Berlin 2002.

DEPARTMENT OF MATHEMATICS

BU-ALI SINA UNIVERSITY

HAMEDAN

IRAN

E-mail: h.samea@basu.ac.ir 